\title{
Simulation and evaluation of FES induced walking
}

\author{
D. van de Belt, H.J. Grootenboer, H.F.J.M. Koopman \\ Laboratory for Biomedical Engineering, Department of Mechanical Engineering, \\ University of Twente \\ P.O.Box 217, 7500 AE Enschede, Netherlands
}

\begin{abstract}
A computermodel is developed for the simulation of FES induced walking. The equations of motion for a 8 segments model are derived and solved with the direct dynamics method, i.e. the input to the model are the joint moments or muscle forces and the output is the movement of the system. Constraint equations are used to reduce the set of system equations. A quasi-Newton optimization method is used to find the optimal combination of stimulated muscles and use of orthoses. The emphasis in this paper is on the development of the mathematical model.
\end{abstract}

\section{INTRODUCTION}

The aim of the project Mobility Restoration at the University of Twente, is the development of a new hybrid system for paraplegic patients. To know more about walking with such a system, a computermodel for the simulation of FES induced walking is developed. With this model we hope to get more insight in this way of walking and to find the optimal combination of stimulation patterns and use of orthoses for a paraplegic patient to walk.

The model will be built up in phases. With the present state of the model a simple case will be worked out, while the results will be compared with the results of measurements, carried out in our laboratory. In the future, the model will be used to search for stimulation patterns and use of orthoses to find alternative walking strategies for paraplegic patients.

\section{METHODS}

\section{A. Model}

The model consists now of eight segments (Fig. 1): two complete three segment lower limbs, the head, arms and trunk as one segment (HAT) and one segment for the pelvis. The pelvis has six degrees of freedom: three translations and three rotations; both hips have three degrees of freedom

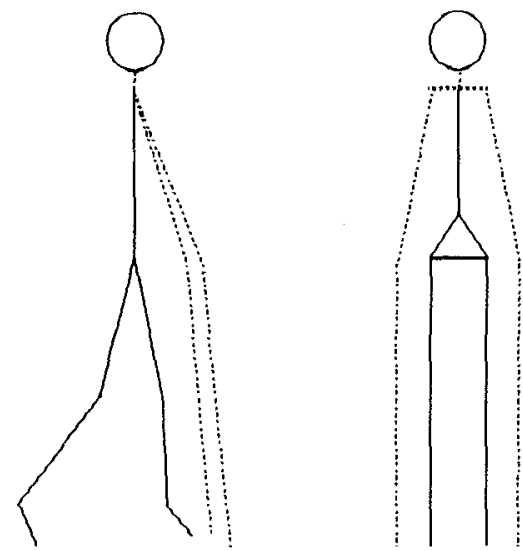

Fig. 1. The segments of the model (scgments with dotted lines will be added in future)

(flexion/extension, ab/adduction and endo/exorotation), just as the connection between HAT and pelvis has and each ankle and knee has one degree of freedom (only flexion / extension). In total there are 19 degrees of freedom. With the symbol manipulation program AUTOLEV [1] the equations of motion are determined. The set of 19 equations of motion is reduced by incorporating the kinematic constraints. E.g. the constraint for the single stance fase, that the foot should be in contact with the floor, yields three constraint equations and reduces the order of the system equation by three. A stiff knee reduces the system matrix by one order.

With:

M: systemmatrix

$\varphi$ : vector of joint rotations

f: vector of forces

C: transformation matrix $\mathrm{C} ; \quad \dot{\varphi}_{\text {tot }}-\mathrm{C} \dot{\varphi}_{\text {indep }}$

and: $\quad M^{*}-C^{T} M C$ and $f^{*}=C^{T}\left(f-M \dot{C} \dot{\varphi}_{\text {indep }}\right)$

the reduced system equation is: $\quad M^{*} \ddot{\varphi}_{\text {indep }}-f^{*}$ 


\section{B. Solving the equations}

The problem solved here is a direct dynamics problem; in this type of problem the forcing functions applied to a mechanical system are known, while the resulting movement has to be determined. This is in contradiction with the inverse dynamic problem, in which the movement of a mechanical system is specified and the forcing functions causing that movement, have to be determined [2]. An important reason to choose the joint moments as the input of the system, is the posibility to take into account an on/off pattern of stimulation.

To solve the equations of motion, not only the joint moments have to be known but also the initial values of the joint rotations and rotational velocities. With a Kutta-Merson integration scheme the movement of the body, due to the joint moments, can be calculated.

The joint restrictions are dealt with by introducing springs at the joint. During the integration proces, the joint restrictions are checked at every time-step, and, if necessary, a spring-force between the two segments will be applied.

Full knee extension is modelled with the use of the conservation of moment of momentum. The same type of equation is used to model heel contact.

Not only the initial position of the body is prescribed, but also the final condition is. In case a full step has to be made, the final joint rotations and velocities are equal to the initial joint rotations and velocities. But first a simpler case will be used: with fully supported arms only one foot will be moved forward while the other foot remains at the same place.

\section{OPTIMIZATION}

To find the joint moments which generate not just a movement but a walking pattern that will lead to the desired end condition, an optimization scheme is needed. The joint moments are taken as control variables in the optimization proces, in which an object function has to be optimized. Because artificial stimulation of the muscles fatigues the muscles more than voluntary stimulation [3], the choice was to minimize the fatigue. As a first step, this fatigue will be represented by the summation of the square of the joint moments.

For the optimization, optimal control is used and a first order gradient algorithm [4]. Fig. 2 gives a schematic representation of the model. To fulfill the final conditions of the joint rotations, the values of these variables at $t=t_{\text {final }}$ are used to get a starting value for the so-called adjoint equations. The backwards integration of the adjoint equations leads to a new estimation of the controls, with which the system equation is integrated again.

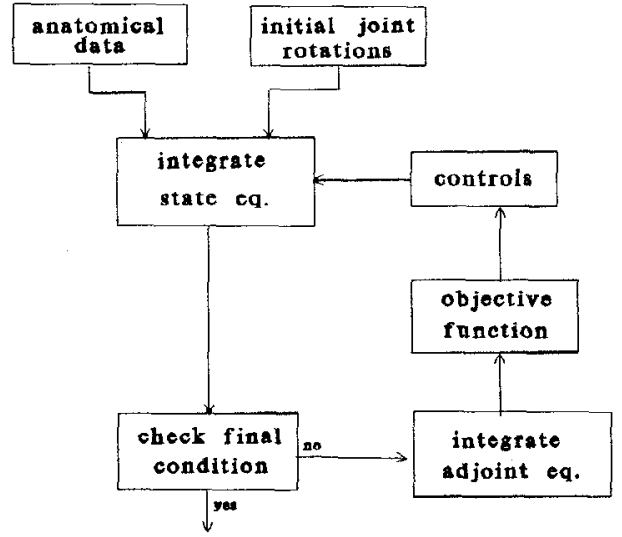

Fig. 2. Schematic representation of the model

This process continues untill the values of the controls and the final constraints do not change anymore within the desired degree of accuracy,

\section{DISCUSSION}

Tests with the integration proces showed that this part is working and that it is possible to carry out a step. Yamaguchi [3] also tried to generate a walking pattern, with the use of dynamic programming. With this method a simple optimization process would take more than a year CPU calculating time. Regarding the calculating time so far, the method of optimization used here shows better expectations to remain within a reasonable amount of CPU time.

The first step will be to take only four controls (moment and two forces at the pelvis and one hip moment) to get an idea about the problem. Next steps concerning the mathematical model will be:

- more controls taken into account

- including muscle models; then the controls can be the stimulation patterns instead of the joint moments

- including separate segments for the arms and walking aids.

\section{REFERENCES}

[1] D.B. Schaechter, D.A. Levinson and T.R. Kane, Autolev user's manual, OnLine dynamiss, Inc., USA, 1991

[2] C.L. Vaughan, J.G. Hay and J.G. Andrews, "Closed loop problems in biomechanics. Part 1 - A classification system", $J$ Biomechanics, vol. 13, pp. 361-368, 1980

[3] G.T. Yamaguchi, Feasibility and conceptual design of functional neuromuscular stimulation systems for the restoration of natural gait to paraplegics based on dynamic musculoskeletal models, dissertation, august, 1989

[4] A.E. Bryson and Y-C Ho, Applied Optimal Control, John Wiley \& Sons, NY, 1975 